University of Wollongong

Research Online

Australian Institute for Innovative Materials -

Papers

Australian Institute for Innovative Materials

2012

Exchange bias in a nanocrystalline hematite/permalloy thin film investigated with polarized neutron reflectometry

David Laurence Cortie

University of Wollongong, dlc422@uowmail.edu.au

K. -W Lin

National Chung Hsing University, Taiwan

H. - F. Hsu

National Chung Hsing University, Taiwan

X L. Wang

University of Wollongong, xiaolin@uow.edu.au

$M$ James

ANSTO

See next page for additional authors

Follow this and additional works at: https://ro.uow.edu.au/aiimpapers

Part of the Engineering Commons, and the Physical Sciences and Mathematics Commons

Research Online is the open access institutional repository for the University of Wollongong. For further information contact the UOW Library: research-pubs@uow.edu.au 


\title{
Exchange bias in a nanocrystalline hematite/permalloy thin film investigated with polarized neutron reflectometry
}

\begin{abstract}
We investigated a hematite $\alpha$-Fe203/permalloy Ni80Fe20 bilayer film where the antiferromagnetic layer consisted of small hematite grains in the 2 to $16 \mathrm{~nm}$ range. A pronounced exchange bias effect occurred below the blocking temperature of $40 \mathrm{~K}$. The magnitude of exchange bias was enhanced relative to reports for identical compounds in large grain, epitaxial films. However, the blocking temperature was dramatically reduced. As the Néel temperature of bulk $\alpha-\mathrm{Fe} 203$ is known to be very high ( $860 \mathrm{~K})$, we attribute the low-temperature onset of exchange bias to the well-known finite-size effect which suppresses the Morin transition for nanostructured hematite. Polarized neutron reflectometry was used to place an upper limit on the concentration and length scale of a layer of uncompensated moments at the antiferromagnetic interface. The data were found to be consistent with an induced magnetic region at the antiferromagnetic interface of $0.5-1.0 \mu \mathrm{B}$ per Fe atom within a depth of 1-2 $\mathrm{nm}$. The field dependence of the neutron spin-flip signal and spin asymmetry was analyzed in the biased state, and the first and second magnetic reversal were found to occur by asymmetric mechanisms. For the fully trained permalloy loop, reversal occurred symmetrically at both coercive fields by an in-plane spin rotation of ferromagnetic domains.
\end{abstract}

\section{Keywords}

bias, investigated, polarized, neutron, reflectometry, nanocrystalline, hematite, permalloy, thin, exchange, film

\section{Disciplines}

Engineering | Physical Sciences and Mathematics

\section{Publication Details}

Cortie, D. L., Lin, K., Hsu, H., Wang, X. L., James, M., Fritzsche, H., Bruck, S. \& Klose, F. (2012). Exchange bias in a nanocrystalline hematite/permalloy thin film investigated with polarized neutron reflectometry. Physical Review B: Condensed Matter and Materials Physics, 86 (5), 054408-1-054408-10.

\section{Authors}

David Laurence Cortie, K. -W Lin, H. - F. Hsu, X L. Wang, M James, H Fritzsche, S Bruck, and F Klose 


\title{
Exchange bias in a nanocrystalline hematite/permalloy thin film investigated with polarized neutron reflectometry
}

\author{
D. L. Cortie, ${ }^{1,2, *}$ K.-W. Lin, ${ }^{3}$ C. Shueh, ${ }^{3}$ H.-F. Hsu, ${ }^{3}$ X. L. Wang, ${ }^{1}$ M. James,${ }^{2,5}$ H. Fritzsche, ${ }^{4}$ S. Brück, ${ }^{2,5}$ and F. Klose ${ }^{2, \dagger}$ \\ ${ }^{1}$ The Institute for Superconducting and Electronic Materials, The University of Wollongong, Wollongong, New South Wales 2522, Australia \\ ${ }^{2}$ Australian Nuclear Science and Technology Organisation, Lucas Heights, New South Wales 2234, Australia \\ ${ }^{3}$ Department of Materials Science and Engineering, National Chung Hsing University, Taichung 402, Taiwan \\ ${ }^{4}$ National Research Council Canada, Canadian Neutron Beam Centre, Chalk River Laboratories, Bldg. 459, Chalk River, \\ Ontario, KOJ 1 JO Canada \\ ${ }^{5}$ The University of New South Wales, Sydney, New South Wales 2052, Australia \\ (Received 28 March 2012; revised manuscript received 17 June 2012; published 7 August 2012)
}

\begin{abstract}
We investigated a hematite $\alpha-\mathrm{Fe}_{2} \mathrm{O}_{3} /$ permalloy $\mathrm{Ni}_{80} \mathrm{Fe}_{20}$ bilayer film where the antiferromagnetic layer consisted of small hematite grains in the 2 to $16 \mathrm{~nm}$ range. A pronounced exchange bias effect occurred below the blocking temperature of $40 \mathrm{~K}$. The magnitude of exchange bias was enhanced relative to reports for identical compounds in large grain, epitaxial films. However, the blocking temperature was dramatically reduced. As the Néel temperature of bulk $\alpha-\mathrm{Fe}_{2} \mathrm{O}_{3}$ is known to be very high $(860 \mathrm{~K})$, we attribute the low-temperature onset of exchange bias to the well-known finite-size effect which suppresses the Morin transition for nanostructured hematite. Polarized neutron reflectometry was used to place an upper limit on the concentration and length scale of a layer of uncompensated moments at the antiferromagnetic interface. The data were found to be consistent with an induced magnetic region at the antiferromagnetic interface of 0.5-1.0 $\mu_{B}$ per Fe atom within a depth of 1-2 nm. The field dependence of the neutron spin-flip signal and spin asymmetry was analyzed in the biased state, and the first and second magnetic reversal were found to occur by asymmetric mechanisms. For the fully trained permalloy loop, reversal occurred symmetrically at both coercive fields by an in-plane spin rotation of ferromagnetic domains.
\end{abstract}

DOI: 10.1103/PhysRevB.86.054408

PACS number(s): 75.70.Cn, 75.70.-i, 75.75.-c, 64.60.an

\section{INTRODUCTION}

Hematite, $\alpha-\mathrm{Fe}_{2} \mathrm{O}_{3}$, is a naturally abundant oxide on Earth and Mars and has been one of the most studied antiferromagnets. ${ }^{1-3}$ A thorough understanding of bulk hematite's exotic magnetic properties such as anisotropic super-exchange, ${ }^{4,5}$ low anisotropy, ${ }^{6}$ and unusual spin structure $^{7}$ was already achieved by the mid-1960s. ${ }^{1}$ More recently, there has been renewed interest in hematite systems at nanometer dimensions for applications in biomedical nanoparticles, ${ }^{8,9}$ spin valves, ${ }^{10-12}$ and chemical sensors. ${ }^{13}$ Based on the work of Dzyaloshinsky ${ }^{4}$ and Moriya, ${ }^{5}$ the magnetic structure of hematite can be explained due to anisotropic super-exchange of the Dzyaloshinsky-Moriya (DM) form, ${ }^{4,5}$ together with nearly zero net-uniaxial anisotropy due to the cancellation of the first-order single ion and dipolar anisotropies. ${ }^{6}$ Together these properties enable spin canting and make hematite the protypical weak ferromagnet, more properly called a canted antiferromagnet. ${ }^{1}$ The lowtemperature reversal in the sign of the uniaxial anisotropy, ${ }^{14}$ along with the DM interaction, explains the magnetic phase diagram of hematite which contains an extra transition between two antiferromagnetic states via temperature-driven spin-flop at the Morin transition. ${ }^{7}, 15$ In addition to a typical spin-flop transition, where the moments reorientate from perpendicular to parallel to the $c$ axis, ${ }^{7}$ this point also corresponds to the vanishing of the canted weak-ferromagnetic behavior which only appears in the high-temperature regime. ${ }^{15}$ Recent experimental works have shown that the magnetic properties of nanostructured hematite can deviate dramatically from the bulk case, including a suppressed Morin temperature
$\left(T_{M}\right)$ depending on grain size, geometry, and strain; ${ }^{15-18}$ a field-driven spin-flop that depends on particle volume and geometry, ${ }^{15,19}$ a quantized spin-wave dispersion, ${ }^{20}$ and an altered spin structure leading to exchange bias in natural ores. ${ }^{21}$ In particular, the wide range of temperatures where exchange bias occurs for ferromagnetic/hematite bilayer thin films ${ }^{11,22-24}$ lacks a convincing explanation. The diversity in blocking temperature for chemically identical bilayers implies that the exchange bias relies on a complex interplay of the finite-size effects described above. Work on similar oxide multilayers has also sometimes reported anomalous magnetic moments in the antiferromagnet layers ${ }^{25}$ or double-step hysteresis loops. ${ }^{26}$ This elicits a second possibility that uncompensated spins or foreign phases embedded within the antiferromagnet, such as $\mathrm{Fe}^{25}$ or $\mathrm{Fe}_{3} \mathrm{O}_{4}$ clusters, ${ }^{27}$ may also play a part in the observed exchange bias. To better understand the mechanism for exchange bias, we investigated a nanocrystalline permalloy/hematite bilayer using a depth-sensitive magnetic neutron scattering technique. Polarized neutron reflectometry provided the means to determine the magnetic depth profile and reversal mechanism in the thin-film system to clarify the magnetic contributions of the oxide layer, permalloy, and interface separately.

\section{METHOD}

A dual ion-beam sputtering deposition technique ${ }^{23}$ was used to prepare the $\mathrm{Ni}_{80} \mathrm{Fe}_{20}\left(t_{1} \mathrm{~nm}\right) / \alpha-\mathrm{Fe}_{2} \mathrm{O}_{3}\left(t_{2} \mathrm{~nm}\right)$ bilayer on a $\mathrm{Si}$ substrate that had previously been annealed to give 
a $\mathrm{SiO}_{2}$ surface layer. The deposition was done at room temperature, below the Néel temperature of the $\alpha-\mathrm{Fe}_{2} \mathrm{O}_{3}$ in an oxygen/(argon + oxygen) ratio of $42 \%$, which was found to produce hematite rather than wurtzite. ${ }^{23}$ The crystal structure of the bilayer was characterized using X-ray diffraction (XRD) on a Panalytical X'Pert Pro instrument. A JEOL (JEM-2010) transmission electron microscope (TEM) was used for microstructural analysis. The surface morphology of the $\alpha-\mathrm{Fe}_{2} \mathrm{O}_{3}$ layer was studied ex situ using a NT-MDT Solver Pro-M atomic force microscope (AFM). Magnetic measurements were performed with a Quantum Design PPMS magnetometer using the vibrating sample magnetometer option where the thin film was in-plane field cooled (FC) in $20 \mathrm{kOe}$ from 300 to $5 \mathrm{~K}$. The neutron reflectometry experiments were performed on the D3 reflectometer located at the NRU reactor in Chalk River (Canada), and using the Platypus reflectometer ${ }^{28}$ at the OPAL reactor in Sydney (Australia). D3 operates at a wavelength of $0.237 \mathrm{~nm}$ using a PG monochromator along with a PG filter to reduce the higher-order contributions of the monochromator, whereas Platypus is a time-of-flight reflectometer maintaining a polarized neutron beam in a 0.25 - to $1.3-\mathrm{nm}$ wavelength spectrum. ${ }^{29}$ High-field neutron reflectometry was performed at D3 using the asymmetric magnetic configuration to preserve the neutron polarization. ${ }^{30}$ Polarized neutron reflectometry measures the reflected neutron intensity near a material's critical edge of total reflection. In such a measurement the independent variables are the wavelength and spin state of the incoming neutron, the angle of incidence, the applied field, and the temperature. The intensity of the reflected neutron beam in different spin states is measured as a function of reflected angle and wavelength usually expressed in terms of the scattering vector,

$$
Q_{z}=\frac{4 \pi \sin (\theta)}{\lambda} .
$$

In this work, the geometry was confined to the case of specular reflection where the incident and reflected angle were equal, and the scattering vector was perpendicular to the surface of the film. The incoming neutron was quantized to one of the two possible spin states (notated here as + and - ), and the outgoing neutron was analyzed to determine if neutron spin-flip scattering had occurred. The reflected intensities for each of the four possibilities was notated as $\mathrm{R}_{++}, \mathrm{R}_{--}$, $R_{+-}$, and $R_{-+}$, where the first subscript is the spin state of the incoming neutron and the second refers to the spin state of the outgoing neutron. For a magnetic sample, each of the reflected intensities depends uniquely on $Q_{z}$ through the altered interference conditions for various layers in the thin-film structure, which in turn depend on the nuclear and magnetic depth profile of the sample. In general, the difference between the non-spin-flip intensities $\mathrm{R}_{++}$and $\mathrm{R}_{--}$is termed spin asymmetry and results from magnetic moments directed along the neutron quantization axis (in this work, the direction of the in-plane applied field). Neutron spin-flip (SF) intensity occurs only if some component of the sample magnetization lies perpendicular to the neutron spin, and also to the scattering vector. X-ray and neutron reflected intensities were fitted with a chemical model and magnetic model using the MOTOFIT ${ }^{31}$ and SIMULREFLEC ${ }^{32}$ software packages.

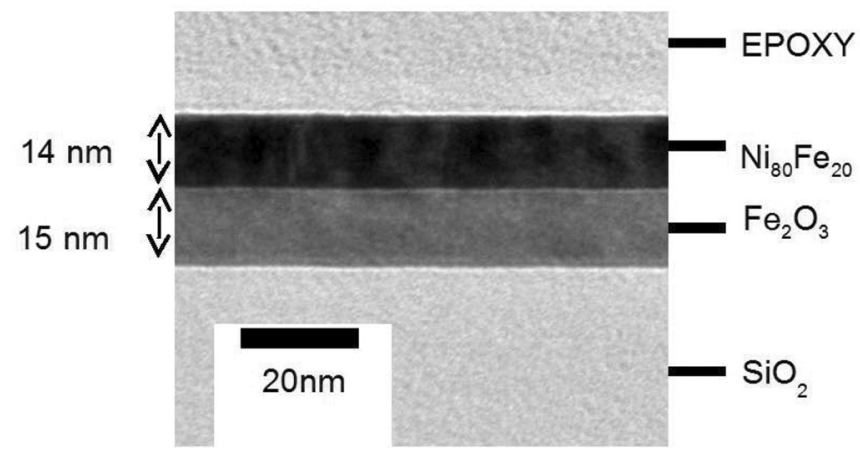

FIG. 1. Cross-sectional TEM image of $\mathrm{Ni}_{80} \mathrm{Fe}_{20} / \alpha-\mathrm{Fe}_{2} \mathrm{O}_{3}$ film showing a uniform film with low interface roughness.

\section{RESULTS AND DISCUSSION}

\section{A. Film structure and morphology}

Figure 1 is the cross-sectional TEM image of the thin film. It shows a clear bilayer structure with flat interfaces, low interfacial roughness $(<2 \mathrm{~nm})$, and layer thicknesses of approximately 14 and $15 \mathrm{~nm}$ for the permalloy and hematite, respectively. The contrast variation within the TEM section of the permalloy layer indicates a degree of varying strain in the film and different crystallite orientations. Figure 2 shows the $\mathrm{x}$-ray reflectometry (XRR) pattern obtained using $\mathrm{Cu}-\mathrm{K} \alpha$ $\mathrm{X}$-rays $(\lambda=0.154 \mathrm{~nm})$ and the best fit using the structural model depicted in the inset. The thicknesses for the $\mathrm{Ni}_{80} \mathrm{Fe}_{20}$ $\left(t_{1}\right) / \alpha-\mathrm{Fe}_{2} \mathrm{O}_{3}\left(t_{2}\right)$ bilayer are found from the XRR to be $t_{1}=13.16 \mathrm{~nm}$ and $t_{2}=14.56 \mathrm{~nm}$. These global values agree

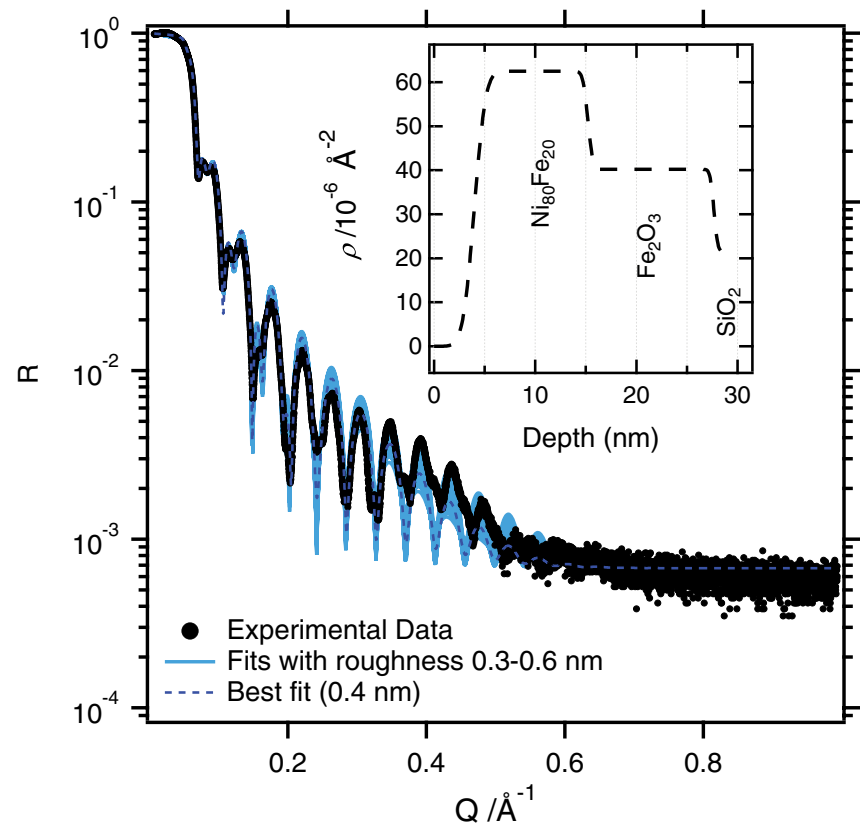

FIG. 2. (Color online) X-ray reflectometry with $\mathrm{Cu}-\mathrm{K}_{\alpha}(0.154-$ $\mathrm{nm})$ radiation. Black points are experimental data. The dotted line is the best fit to the data. The shaded area encompasses a series of fits to the data using different interfacial roughnesses for the buried hematite interface in the range $0.3-0.6 \mathrm{~nm}$. The inset shows the scattering length density model used to fit the data. 

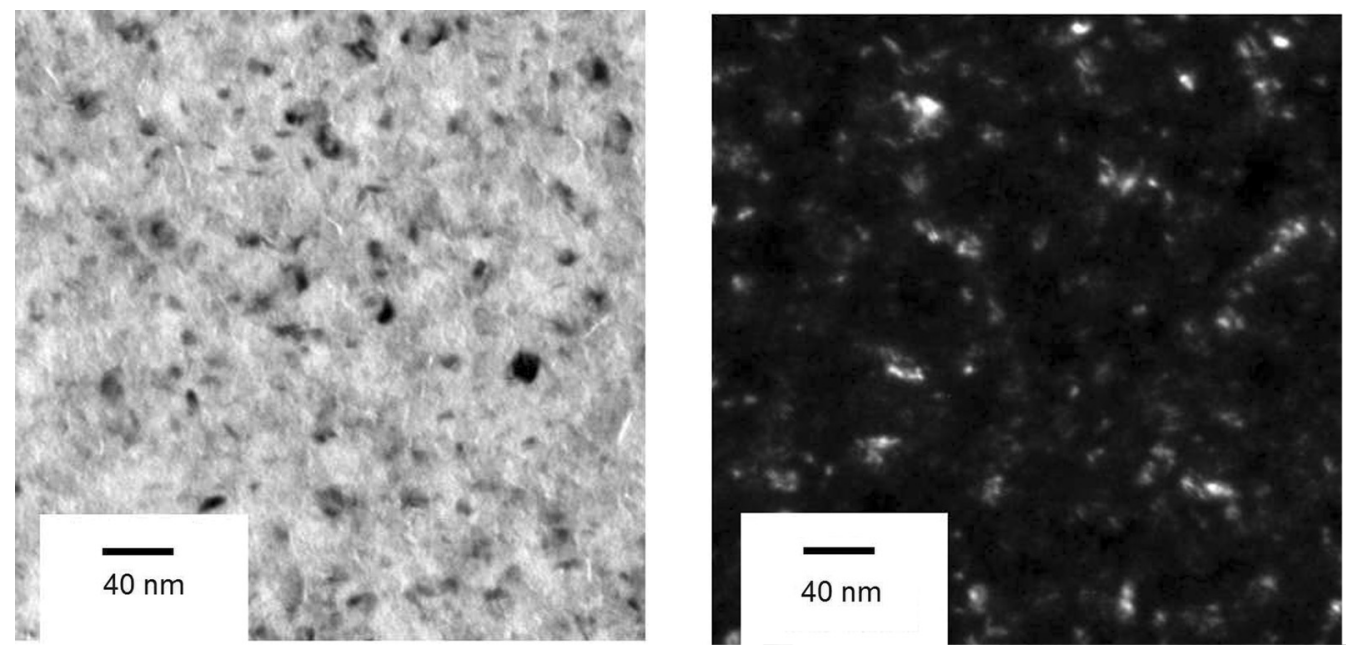

FIG. 3. (a) Bright-field (left) and (b) dark-field TEM (right) micrographs taken of the hematite surface without permalloy deposition showing a highly nanocrystalline hematite material with grain sizes in the 2 to $16 \mathrm{~nm}$ range.

adequately with the local thicknesses found from the TEM cross section. The chemical composition and number density of $\mathrm{Fe}, \mathrm{O}$, and $\mathrm{Ni}$ ions in the $\mathrm{X}$-ray model are in agreement with the values for bulk hematite and permalloy. The interfacial roughness can be quantified from the XRR fits as $0.4 \pm 0.2 \mathrm{~nm}$. Figures 3(a) and 3(b) are the bright-field and dark-field TEM images, respectively, taken ex situ for the hematite surface, showing a highly nanocrystalline material with small grain dimensions ranging between 2 and $16 \mathrm{~nm}$. The scarcity of bright spots in the dark-field image indicates that relatively few of the hematite grains simultaneously meet the diffraction condition suggesting an untextured arrangement of hematite grain orientations. Figure 4 shows a histogram of the grain size distribution in the hematite layer found by performing a particle analysis of the dark-field TEM using the IMAGEJ software suite. ${ }^{33}$ The mean grain area is found to be $13 \mathrm{~nm}^{2}$. Ex situ AFM of the top $\alpha-\mathrm{Fe}_{2} \mathrm{O}_{3}$ interface was conducted by depositing a trial $\alpha-\mathrm{Fe}_{2} \mathrm{O}_{3}$ layer, without depositing the subsequent $\mathrm{Ni}_{80} \mathrm{Fe}_{20}$ layer, with the result shown in Fig. 5. A $\mathrm{rms}$ roughness of $0.2 \mathrm{~nm}$ was found across a $1-\mu \mathrm{m}$ region of the sample, which is in agreement with the value for the buried interface derived from the XRR fit. Figure 6 is the electron

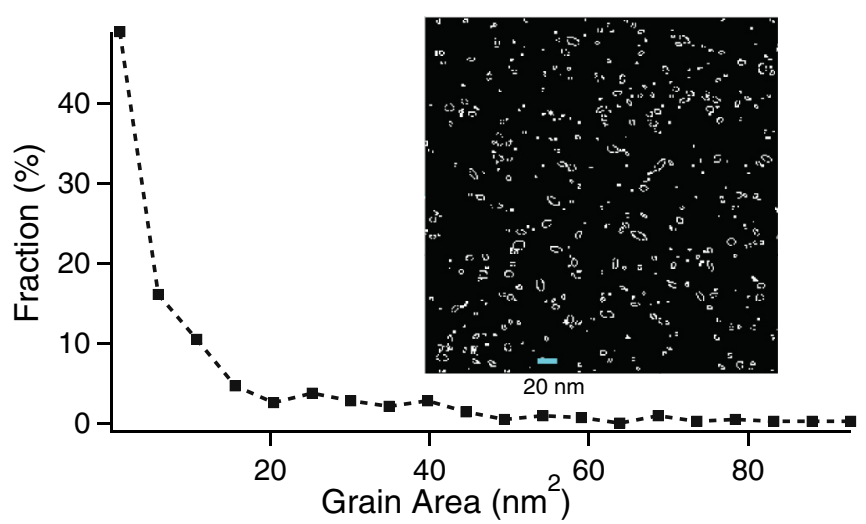

FIG. 4. Grain-size distribution derived from a 1000-nm-square area dark-field TEM image using threshold particle analysis. (Inset) Analyzed particles. and $\mathrm{x}$-ray diffraction pattern for the thin film. The electron diffraction pattern gives lattice constants of $a=5.02 \AA$ and $c=13.93 \AA$ which are slightly altered relative to the bulk hematite values of $a=5.04 \AA$ and $c=13.77 \AA$. The weak peaks in the $\mathrm{x}$-ray diffraction in Bragg-Brentano geometry in Fig. 6 are due to the small thickness of the film combined with the lack of a strong crystalline texture, suggesting that both layers are predominantly nanocrystalline, although the permalloy has a weak (111) columnar texture. ${ }^{23}$ The XRD result is similar to other films studied elsewhere using similar deposition techniques. ${ }^{34}$ Although there is a sharp Bragg peak in the vicinity of the $\alpha-\mathrm{Fe}_{2} \mathrm{O}_{3}$ (104) reflection, this is attributed to secondary scattering leading to a virtual $\mathrm{Si}(200)$ peak. $^{35}$

\section{B. Room-temperature magnetic properties}

Figure 7 is the magnetic hysteresis of the sample measured at $300 \mathrm{~K}$. At room temperature, the bilayer system shows a low coercive field which is typical for a single permalloy film in the unbiased state. Previous groups have reported a significant exchange bias field and fourfold anisotropy at $300 \mathrm{~K}$ for epitaxial $\mathrm{Ni}_{80} \mathrm{Fe}_{20} / \alpha-\mathrm{Fe}_{2} \mathrm{O}_{3}$ films. ${ }^{22}$ These features are not seen for our small, randomly orientated granular system. Whereas the previous group reported exchange bias of up

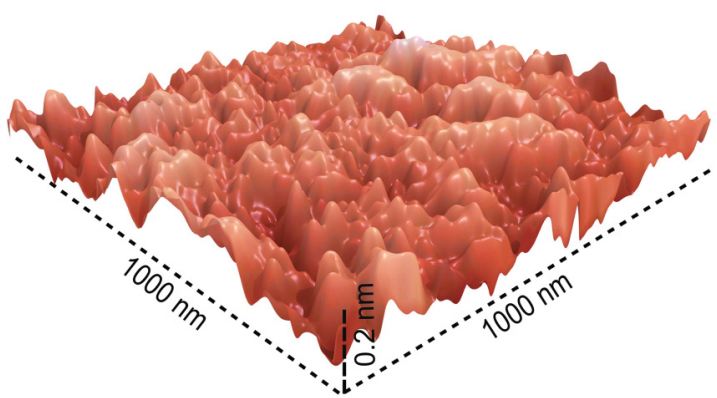

FIG. 5. (Color online) Atomic force microscope image of a typical $\alpha-\mathrm{Fe}_{2} \mathrm{O}_{3}$ underlayer before $\mathrm{Ni}_{80} \mathrm{Fe}_{20}$ deposition. The rms roughness value is $0.2 \mathrm{~nm}$ before permalloy deposition. 


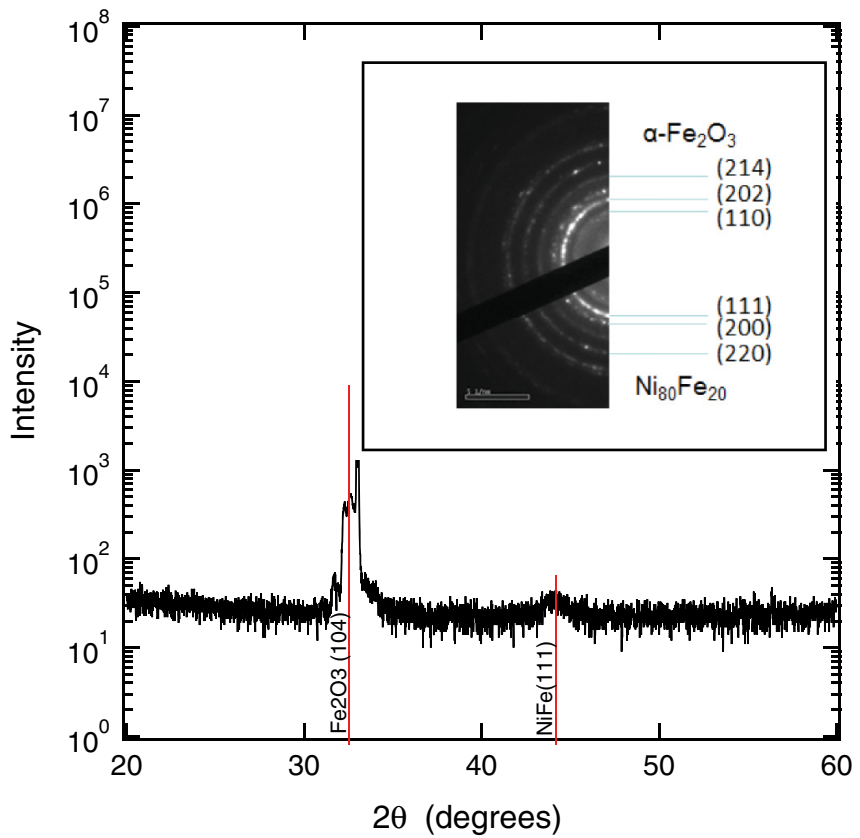

FIG. 6. (Color online) X-ray diffraction pattern in BraggBrentano geometry using $\mathrm{Cu}-\mathrm{K} \alpha$ radiation. The inset shows the electron diffraction pattern.

to 40 Oe for their system, the maximum loop shift for our as-prepared sample is $+5.0 \mathrm{Oe}$, which is smaller than the zero-point error in our PPMS system (10 Oe) and, therefore, must be considered to lie within experimental uncertainty. Magnetic field annealing (MFA) by heating the sample to $360 \mathrm{~K}$, and applying a 20-kOe field for $60 \mathrm{~min}$, did not appreciably alter the loop shift on cooling to $300 \mathrm{~K}$, although the coercivity was decreased.

Figure 8 is the polarized neutron reflectometry patterns for the bilayer at $5 \mathrm{kOe}$ and $300 \mathrm{~K}$. Good fits to the data can be generated using the parameters listed in Tables I and II, where the differences between the two models are discussed in detail later. In the simpler model, described in Table I, hematite has a zero moment, whereas a large $0.96 \mu_{B}$ per formula unit (FU) moment is found in the ferromagnetic permalloy consistent with the bulk saturation value known for permalloy.

To check if a weak ferromagnetic moment throughout the hematite layer could be enhanced in high magnetic fields,

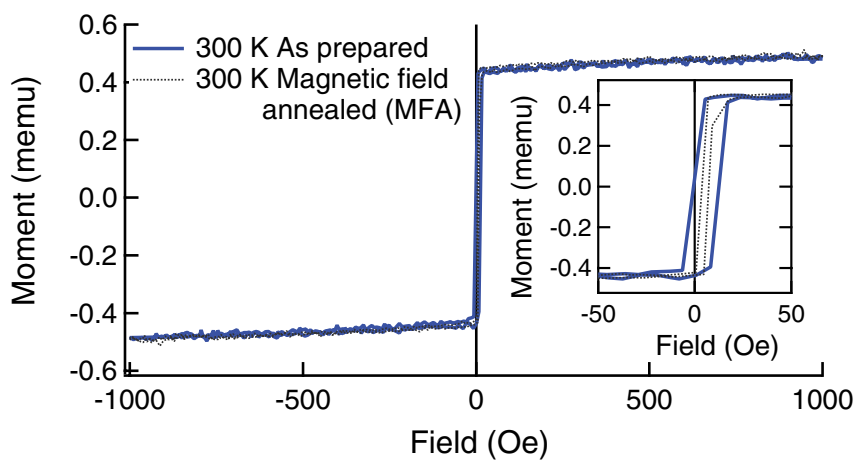

FIG. 7. (Color online) Room-temperature magnetometry. (Inset) Enlargement of the low-field region.

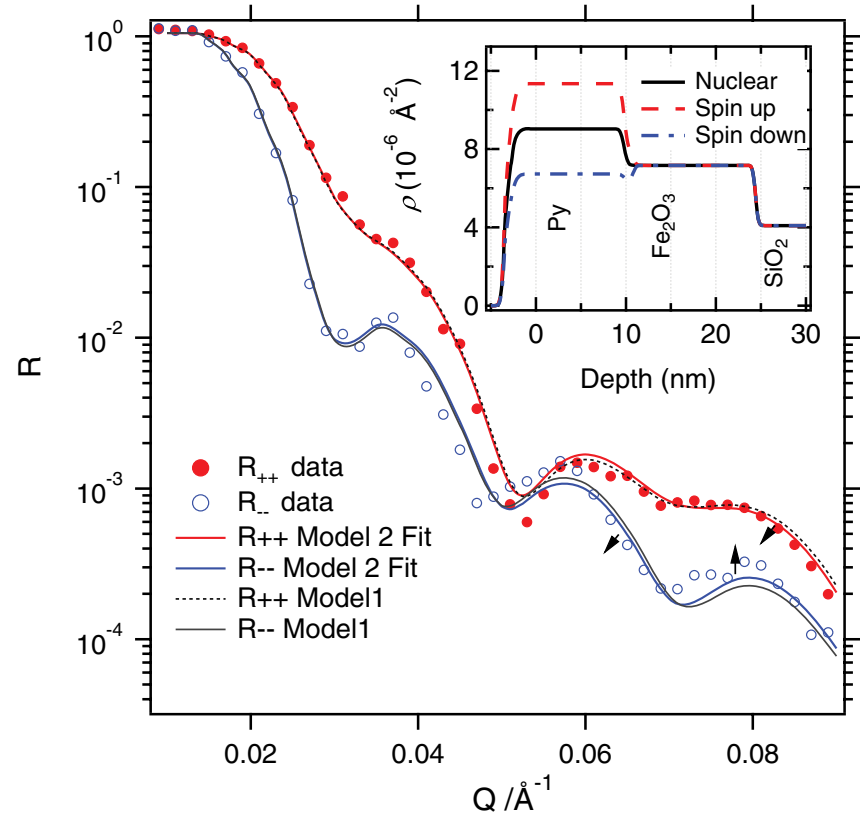

FIG. 8. (Color online) Room-temperature polarized neutron reflectometry patterns with fits according to model 1 and model 2 with parameters $x=1.0 \mathrm{~nm}, M=0.75 \mu_{B}$ per Fe atom, as discussed in the text. Error bars lie within symbols. The arrows indicate the trend for how the two models differ.

we collected a polarized neutron reflectometry pattern at $30 \mathrm{kOe}$ (not shown) at the same temperature. The high-field measurement at $30 \mathrm{kOe}$ was found to be similar to the low-field measurement in that both could be best fitted using the model of near-zero moment in the bulk part of the hematite. In this way, the PNR shows that, even at high fields, the nanoscale hematite thin film does not have a bulk magnetic moment that is detectable by neutron reflectometry $\left(<0.05 \mu_{B}\right.$ per $\mathrm{Fe}$ atom) which is consistent with the low moment known for bulk and nanoparticle hematite in the weak ferromagnetic state. ${ }^{15,19}$ These measurements also rule out the possibility of sizable magnetic impurity clusters and stand in contrast to a recent report on an iron oxide thin film which found an anomalous $0.5 \mu_{B}$ moment per Fe atom in a nominally antiferromagnetic layer. ${ }^{25}$ Despite the near-zero moment in the bulk of the hematite, a more complex magnetic structure which includes a net moment concentrated in the interfacial region of the hematite yields a better fit to the data with the range of parameters in Table II.

Figure 8 compares the fits using the two models, where, in both cases, the same nuclear scattering-length density profile was adopted from the layer thicknesses, the compositions and interface roughnesses derived from the x-ray reflectometry and cross-sectional TEM results, and only the magnetic moment was allowed to vary as a free parameter. Visually, the effect of including the uncompensated interface moment is clearly detectable, even on a logarithmic scale, and results in an improved fit in the $Q_{z}$ region between $0.06 \AA^{-1}$ and $0.09 \AA^{-1}$. Figure 9 shows the residual error $r_{U}$ for a range of fits with different magnitudes of induced magnetism at the hematite interface, where $r_{U}$ has been calculated by the SIMULREFLEC software $^{32}$ using the formula $r_{U}=\sum\left[\frac{R_{\exp }\left(Q_{z}\right)-R_{\text {calc }}\left(Q_{z}\right)}{R_{\exp }\left(Q_{z}\right)+R_{\text {calc }}\left(Q_{z}\right)}\right]^{2}$ to 
TABLE I. Best-fit parameters to polarized neutron reflectivity data at $300 \mathrm{~K}$ for model 1, with only one magnetized layer.

\begin{tabular}{lccc}
\hline \hline Layer & Thickness (nm) & Roughness (nm) & $\mu_{B}$ per formula unit \\
\hline $\mathrm{Ni}_{80} \mathrm{Fe}_{20}$ & 13.15 & 1.03 & 0.96 \\
$\mathrm{Fe}_{2} \mathrm{O}_{3}$ & 14.58 & 0.46 & 0 \\
$\mathrm{SiO}_{2}$ & 98 & 0.35 & 0 \\
$\mathrm{Si}$ & Infinite & 0.5 & 0 \\
\hline \hline
\end{tabular}

sum the differences between the experimental and fitted points across the entire $Q_{z}$ range for the reflectivities in both spin channels. For the high-field and room-temperature data, the minimum error clearly occurs at a nonzero interfacial moment, showing that the overall error of the fit can be lowered by including an induced moment in the hematite within $1 \mathrm{~nm}$ of the interface, with a magnitude of $0.5-1.0 \mu_{B}$ per Fe atom for the $300 \mathrm{~K}, 30 \mathrm{kOe}$ data. According to current understanding of exchange bias, the interfacial exchange coupling between ferromagnetic and antiferromagnetic spins, along with the external field, can potentially overcome the antiferromagnetic exchange forces and lead to an induced polarization of the antiferromagnet, where $1-15 \%$ of the per-site AF moment can be polarized near the interface due to the formation of antiphase domains ${ }^{36}$ or spin canting. ${ }^{37}$ Hematite has $4 \mu_{B}$ per Fe atom, ${ }^{18}$ and, therefore, the experimentally fitted magnetic moment is consistent with the polarization predicted by such models. Precise measurement of the weak induced moments at an antiferromagnetic interface remains challenging using polarized neutron reflectometry. ${ }^{38}$ However, there is a growing body of evidence using polarized neutron reflectometry ${ }^{39-41}$ and other experimental techniques ${ }^{42-44}$ that confirms these net moments exist in a wide range of exchange bias systems, although the origin, length scale, and magnetic concentration still remain under debate. To illustrate this point, Fig. 10 is the contour plot of the dependency of the relative fit error $\Delta r_{U}=\frac{r_{U}(M, x)-r_{0}}{r_{0}}$, where $r_{U}(M, x)$ is the fit residuum calculated for a combination of $x$ interface thickness $(\mathrm{nm})$ and $M$ magnetic moment $\left(\mu_{B}\right.$ per $\mathrm{Fe}$ ) and $r_{0}$ is the lowest error taken from the point at $M=0.75 \mu_{B}$ per $\mathrm{Fe}$ and $x=1.0 \mathrm{~nm}$. From the roughly triangular area enclosed in the first contour, it is clear that a range of different combinations of induced layer thickness $(x)$ and magnetic moment give a good fit where the relative error lies in the $0-5 \%$ range, which can be taken as reasonable estimate of uncertainty. ${ }^{45}$ From this region, it appears that a range of fits with low error can be generated where $x$ is in the range $0.75-2.1 \mathrm{~nm}$ and the maximum magnetic moment is roughly proportional to $\frac{1.0}{x}\left(\mathrm{~nm} \mu_{B}\right)$. The upper limit of a 2-nm induced moment seems to be of surprisingly long range compared to the other induced moments. ${ }^{43}$ On the other hand, a recent theoretical result showed that the DzyaloshinskyMoriya interaction should enhance the interfacial moment in exchange bias systems with canted antiferromagnets, ${ }^{37}$ and, moreover, bulk hematite shows coherent spin canting over multiple atomic monolayers. ${ }^{1,2,15}$ The effect of strong interfacial spin canting was previously inferred from the magnetometry results on similar $\alpha-\mathrm{Fe}_{2} \mathrm{O}_{3}$ films. ${ }^{23}$ In bulk hematite the canting angle is very $\operatorname{small}^{18}\left(\approx 0.1^{\circ}\right)$, but the maximum interface-spin canting angle for the hematite thin film studied here needs to be signifigantly higher $(\approx 7-$ $15^{\circ}$ ) to be consistent with the neutron data, in which case the interfacial antiferromagnetic spins would have a more significant effect on exchange-coupled ferromagnetic spins. Admittedly, neutron reflectometry cannot uniquely determine whether an interfacial moment originates from noncollinear spin canting or a small percentage of collinear uncompensated spins, since it measures only the net moment. However, in this case, it provides insight that it is iron-oxide spins, rather than permalloy spins, that behave differently, because altering the nuclear scattering-length density of the interlayer worsened the fit. This suggests that it is unlikely that chemical interdiffusion or formation of foreign magnetic interface phases could explain the experimental observations. ${ }^{26}$ Moreover, decreasing or increasing the permalloy moment within 1-2 nm of this region increased the error. Despite the probable existence of an induced interface moment, significant exchange bias is not seen in the magnetic hysteresis. The best explanation for this is that, at $300 \mathrm{~K}$, our sample lies above the Morin temperature which occurs at $<100 \mathrm{~K}$ for nanocrystallites, whereas the other group stated that, for their strained epitaxial films, this transition is enhanced to $400 \mathrm{~K}^{22}$ Similar suppression of the exchange bias temperature has been reported previously for nanostructured hematite systems. ${ }^{24}$ The absence of exchange bias, even in the presence of an interface moment, is not altogether surprising since the Morin transition is concurrent with a sizable increase in the uniaxial anisotropy ${ }^{6}$ of hematite towards low temperature, which in turn, stabilizes antiferromagnetic order within the small grains against

TABLE II. Best-fit parameters for polarized reflectivity data at $300 \mathrm{~K}$ for model 2, with two magnetic layers, including an induced magnetic region at the hematite interface.

\begin{tabular}{lccc}
\hline \hline Layer & Thickness (nm) & Roughness (nm) & $\mu_{B}$ per formula unit (FU) \\
\hline $\mathrm{Ni}_{80} \mathrm{Fe}_{20}$ & 13.15 & 1.03 & 0.96 \\
$\mathrm{Fe}_{2} \mathrm{O}_{3}$ interface & $0.75 \geqslant x \leqslant 2.1$ & 0.46 & $0.1-2.25$ \\
$\mathrm{Fe}_{2} \mathrm{O}_{3}$ & $(14.58-x)$ & 0 & 0 \\
$\mathrm{SiO}_{2}$ & 98 & 0.35 & 0 \\
$\mathrm{Si}$ & Infinite & 0.5 & 0 \\
\hline \hline
\end{tabular}




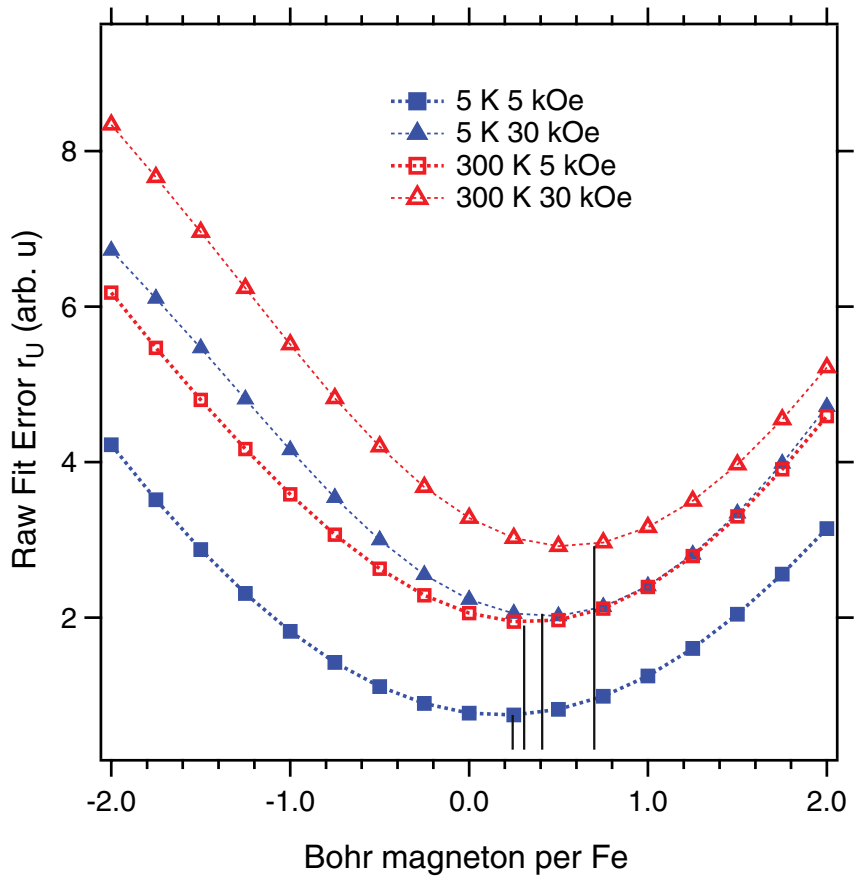

FIG. 9. (Color online) Improvement of neutron fit residuum $\mathbf{r}_{U}$ with altered hematite interface moment at $300 \mathrm{~K}$ and $5 \mathrm{~K}$. Data are the difference between the fit and data calculated in the SIMULREFLEC software for the model with $x=1.0 \mathrm{~nm}$. The vertical black lines denote the fitted position of the minimum error for the connected data set.

superparamagnetic activation, ${ }^{46,47}$ reversal, ${ }^{48}$ and canting. If the magnetocrystalline anisotropy of antiferromagnetic spins is sufficiently low, canted antiferromagnetic spins can be more easily polarized through coupling to ferromagnetic neighbors but concurrently provide less of an energy barrier during reversal. ${ }^{49}$ The uniaxial anisotropy of bulk hematite has a near-zero value at room temperature due to the cancellation of the single-ion and dipolar sources of anisotropy. ${ }^{6,50}$ For nanoparticles of $<10 \mathrm{~nm}$, it is well known that the Morin transition is suppressed, sometimes to below $4 \mathrm{~K},{ }^{15}$ and the case is also true for mesoporous hematite solids. ${ }^{18}$ Our films show a magnetic transition below $100 \mathrm{~K},{ }^{23}$ which could be interpreted as the Morin spin-flop, and is concurrent with the onset of exchange bias.

\section{Low-temperature magnetic properties}

Figure 11 is the temperature dependence of the fully trained magnetic hysteresis starting from lower temperatures. Fully trained means that the sample was field cycled at least 5 times prior to the first measurement. After field cooling in $+20 \mathrm{kOe}$ from $300 \mathrm{~K}$ to $5 \mathrm{~K}$, a strong negative exchange bias of $\mathrm{H}_{\mathrm{EB}}=-200$ Oe appears. The inset of Fig. 11 illustrates that the exchange bias decreases sharply above $10 \mathrm{~K}$ and vanishes by $40 \mathrm{~K}$. A small feature appears in the saturation magnetization near the blocking temperature. In fact, the saturation is almost constant over the $5-300 \mathrm{~K}$ temperature range, corresponding to a value of $800 \pm 30 \mathrm{per} \mathrm{emu} / \mathrm{cm}^{3}$ of permalloy. However, as previously noted, ${ }^{23}$ there is a small downturn in the magnetization below $40 \mathrm{~K}$ followed by a

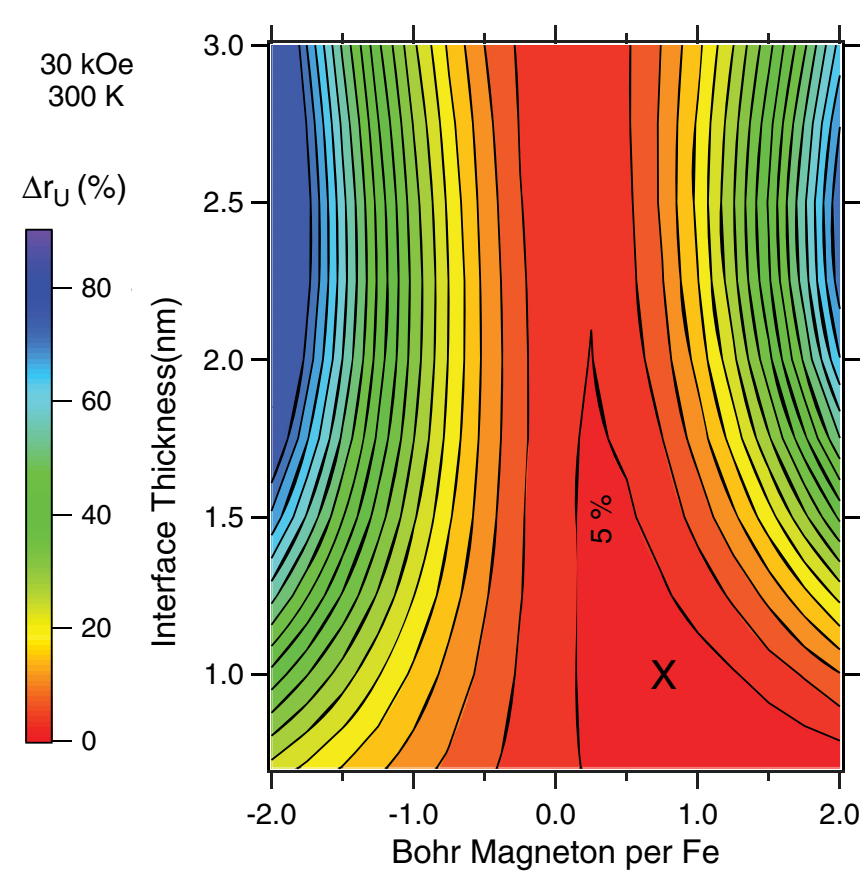

FIG. 10. (Color online) Contour plot of the relative changes in the fit error $\Delta r_{U}=\frac{r_{U}(M, x)-r_{0}}{r_{0}}$ versus the depth and magnitude of the induced moment at the antiferromagnetic hematite interface for the $300 \mathrm{~K}, 30 \mathrm{kOe}$ data set. The roughly triangular area enclosed in the first contour is the range of best-fit parameter combinations. The $\mathrm{X}$ marks the position of the minimal value taken for $r_{0}$.

sharp up-turn towards lower temperatures. The small downturn corresponds to a loss of magnetization of approximately $10 \mathrm{emu} / \mathrm{cm}^{3}$, close to the loss of weak-ferromagnetism occurring for hematite nanograins at the Morin temperature. ${ }^{15}$ Below this kink, there is also an increase towards lower temperatures that was previously attributed to increased spin canting at the interface..$^{23}$ An equally feasible explanation is that a proportion of the smaller hematite grains lie beneath the critical size threshold for the Morin temperature and are trapped in the unblocked weak ferromagnetic state giving the increase of saturation. ${ }^{15}$ These experimental features, and

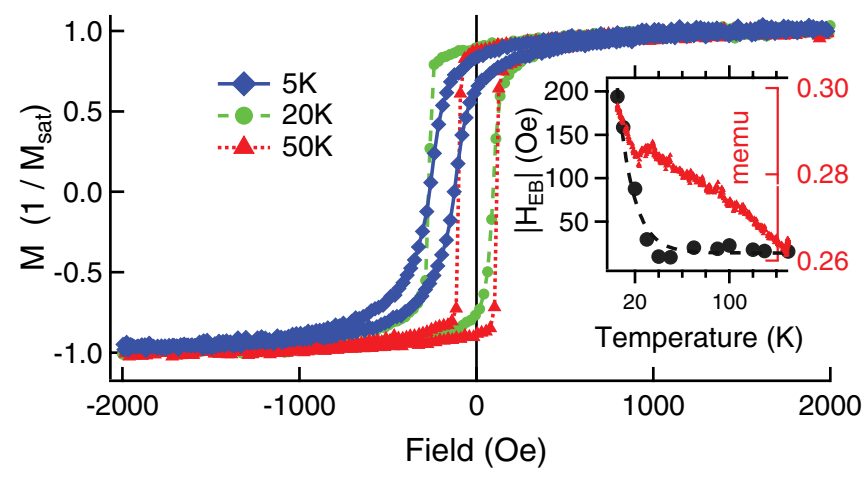

FIG. 11. (Color online) Temperature dependence of fully trained exchange-biased loop after field cooling from $300 \mathrm{~K}$ to $5 \mathrm{~K}$ in $20 \mathrm{kOe}$. (Inset) Comparison of the magnitude of the exchange bias of the film, recorded after field cooling to $5 \mathrm{~K}$ and subsequent heating, with the magnetic moment recorded during cooling in a field of $20 \mathrm{kOe}$. 


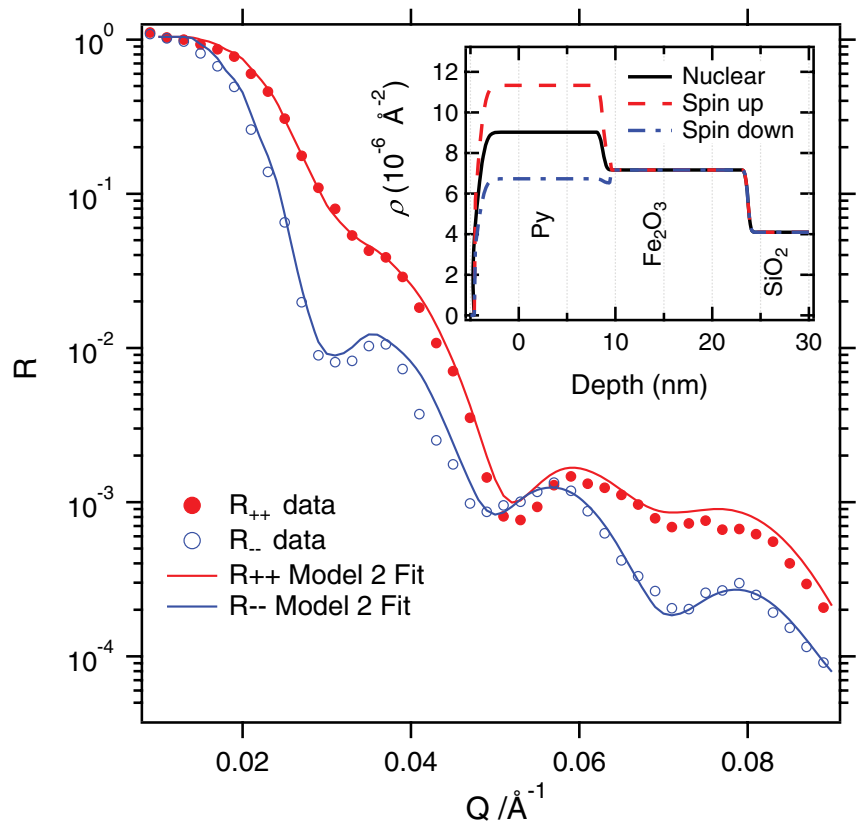

FIG. 12. (Color online) Neutron reflectivities at $5 \mathrm{~K}$ and $5 \mathrm{kOe}$. Error bars lie within symbols. (Inset) Scattering length density profile derived from the fit.

the onset of exchange bias, agree well with the previously published magnetometry data on similar films. ${ }^{23}$ Figure 12 shows the fitted magnetic depth profile for the fully trained system obtained by neutron reflectometry at $5 \mathrm{~K}$ after field cooling in $20 \mathrm{kOe}$. Despite the dramatic alteration of macroscopic magnetic hysteresis due to exchange bias, the experimental magnetic depth profile is similar to that of the room-temperature measurement, suggesting that the bilayer only changes very subtly in the exchange bias state. As for the room-temperature state, the data at low temperature is consistent with a hematite interface moment, although, in this case, the upper limit of the magnitude of the interface moment per $\mathrm{Fe}$ atom for the $5 \mathrm{kOe}$ data is reduced to $\approx \frac{0.2}{x}$ $\left(\mu_{B} \mathrm{~nm}\right)$, where $x$ is the thickness in nanometers. This is understood from the comparison of the fit minima in Fig. 9. The result shows that the magnitude of the uncompensated moment within the antiferromagnetic compound cannot be directly correlated with the strength of exchange bias. The Morin transition to the true antiferromagnetic state would result in the tendency to reduce canting; on the other hand, the remaining uncompensated spins would have an increased anisotropy. ${ }^{6}$ Both factors are necessary for exchange bias. ${ }^{47,49}$ The smaller interfacial moment at low temperature suggests the best explanation for the up-turn in the magnetization beneath the transition temperature is not increased spin canting but rather the interplay of superparamagnetic behavior with the intrinsic weak ferromagnetism of bulk hematite spins in grains too small to undergo the Morin temperature. From the literature on similarly sized hematite nanoparticles, the weak ferromagnetic behavior would give a moment of only $0.5-5 \mathrm{emu} / \mathrm{g},{ }^{15,19}$ corresponding to a maximum of $0.05 \mu_{B}$ per formula unit, which is consistent with the near-zero moment found in the neutron fits.

\section{Magnetic training and reversal mechanism at $5 \mathrm{~K}$}

Figure 13 shows five successive hysteresis loops taken after field cooling to $5 \mathrm{~K}$. Even at this low temperature, the exchange bias appears to be unstable, showing training effects for subsequent reversals. The first loop shows an abrupt, steplike decrease similar to that seen in $\mathrm{Co} / \mathrm{CoO}$ exchange bias systems. ${ }^{51-53} \mathrm{In} \mathrm{Co} / \mathrm{CoO}$ it is reported that all loops after the second are essentially identical, ${ }^{52,54}$ whereas for the hematite system, the subsequent four loops have a similar shape but display systematic differences in coercivity and loop shift. The fourth and subsequent loops are identical within the uncertainties of experimental measurement. The high degree of training even at low temperature may indicate the unavoidable activation of small grains during magnetic reversal, which in turn prevents the usage of a measurement procedure such as the York protocol. ${ }^{47}$ This makes the reproducibility of the measurements closely dependent on the magnetic history and precise measurement sequence used. Numerical models have shown that training can result from an irreversible spin-flop of the antiferromagnetic order in the first reversal, ${ }^{47,54}$ followed by a reversible thermal activation of antiferromagnetic grains on subsequent field cycling for systems with lower antiferromagnetic anisotropy. ${ }^{47}$ Other groups reported that the magnetic reversal mechanism occurring at the first and second reversal points is asymmetric in many exchange bias systems such as $\mathrm{Co} / \mathrm{CoO},{ }^{51-53} \mathrm{Fe} / \mathrm{MnPd}{ }^{55}$ $\mathrm{Fe} / \mathrm{FeF}_{2},{ }^{56}$ and $\mathrm{MnF}_{2} / \mathrm{Fe}^{57}$ This supports the idea that an irreversible change occurs at the antiferromagnetic interface during the first switch, which subsequently affects the ferromagnet's reversal mode. However, there appears to be a divergence in the literature concerning the details for different

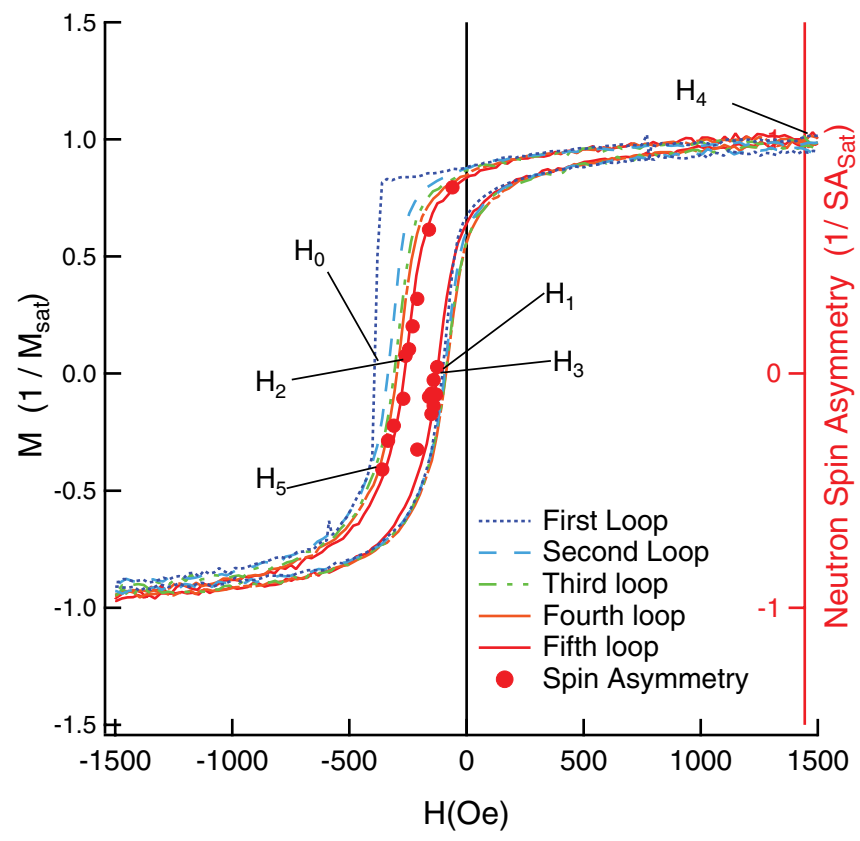

FIG. 13. (Color online) Magnetic training of permalloy/hematite interface at $5 \mathrm{~K}$ after field cooling from $300 \mathrm{~K}$ in $+20 \mathrm{kOe}$. The neutron spin asymmetry (SA) at $q=0.028 \AA^{-1}$ has been plotted over the trained magnetometry loop at $5 \mathrm{~K}$. Both the spin asymmetry and magnetization have been normalized by the value measured at saturation. 
systems, suggesting that the ultimate reversal mechanism may not be universal. Certain groups have reported domain wall motion at the first switching point and some form of spin rotation for the subsequent reversals in $\mathrm{Co} / \mathrm{CoO} .^{51-53}$ However, the opposite seems to be true for $\mathrm{MnF}_{2} / \mathrm{Fe}^{56}$ and $\mathrm{Fe} / \mathrm{MnPd}^{55}$ systems. In many of the papers mentioned, the details regarding the precise level of training, and measurements subsequent to the second reversal, were not explicitly given but the trained behavior was usually inferred from the second switching point (which technically still belongs to the untrained loop). In this work, we deliberately compared the magnetic depth profile derived from PNR at the first and second switching points of the untrained $\left(\mathrm{H}_{0}\right.$ and $\left.\mathrm{H}_{1}\right)$ and trained loops $\left(\mathrm{H}_{2}\right.$ and $\left.\mathrm{H}_{3}\right)$. Short scans at a single angle were used to locate the position of the coercive field, which in each case corresponded to the vanishing of the spin asymmetry. Adequate agreement was found between the magnetometry and neutron spin asymmetry for the sample, as shown in Fig. 13. The magnetic field points where full reflectometry patterns were collected are labeled as $\mathrm{H}_{0-5}$. Figure 14 shows the neutron reflectometry patterns for the coercive fields $\mathrm{H}_{1}$, $\mathrm{H}_{2}$, and $\mathrm{H}_{3}$. A strong trend emerged for the second and subsequent reversals whereby the majority of ferromagnetic magnetic moments pointed at almost $90^{\circ}$ to the field. It is important to note that data sets for all three fields have been overplotted here to emphasize the high degree of symmetry at the left and right coercive fields for the trained loop. Fitting the SF signal gave a moment of $0.96 \mu_{B}$ per formula unit for the permalloy rotated in-plane to an angle of $86 \pm 3^{\circ}$ with respect to the applied field. The fitted magnetization angle for the various fields is summarized in Table III. The

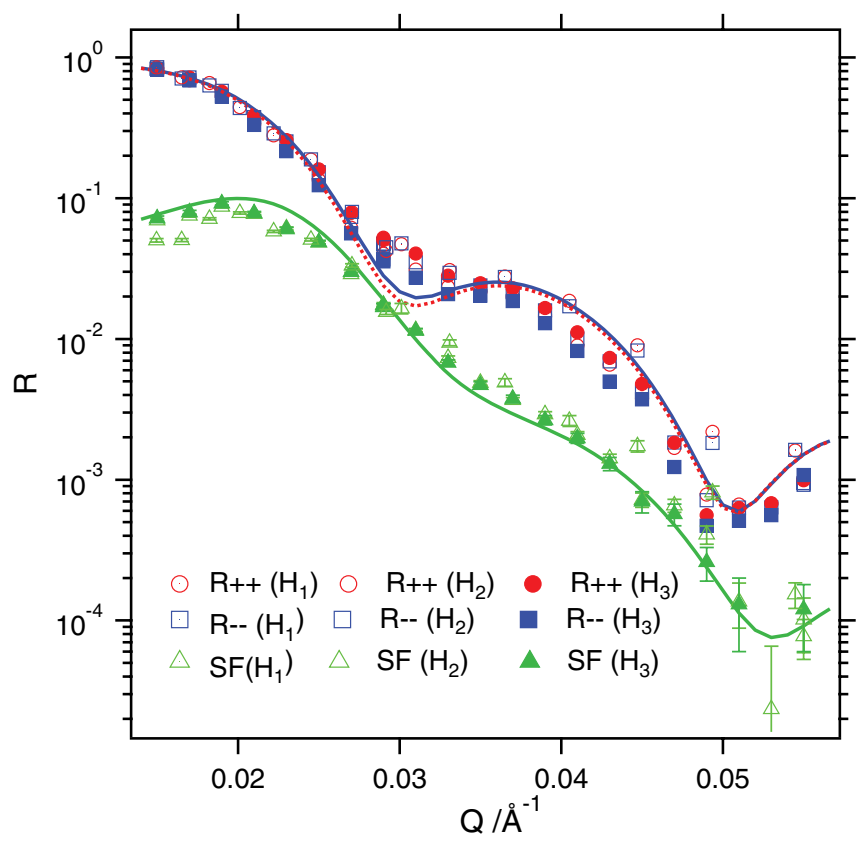

FIG. 14. (Color online) Non-spin-flip and neutron spin-flip reflectivities at points $\mathrm{H}_{1}, \mathrm{H}_{2}$, and $\mathrm{H}_{3}$. The appearance of the neutron spin-flip intensity (green triangles) is due to a large proportion of ferromagnetic spins aligning perpendicularly to the applied field throughout the permalloy layer. The situation is nearly identical at $\mathrm{H}_{1}$ and $\mathrm{H}_{3}$.
TABLE III. Summary of fitted magnetization angles for different fields during magnetic reversal at $5 \mathrm{~K}$ in the trained, field cooled state.

\begin{tabular}{lcc}
\hline \hline Field point & $\mathrm{Ni}_{80} \mathrm{Fe}_{20}$ magnetization per formula unit & Angle \\
\hline $\mathrm{H}_{0}$ & $0.75 \mu_{B}$ & 89 \\
$\mathrm{H}_{1}$ & $0.96 \mu_{B}$ & 87 \\
$\mathrm{H}_{2}$ & $0.96 \mu_{B}$ & 86 \\
$\mathrm{H}_{3}$ & $0.96 \mu_{B}$ & 84 \\
$\mathrm{H}_{4}$ & $0.96 \mu_{B}$ & 0 \\
$\mathrm{H}_{5}$ & $0.9 \mu_{B}$ & 74 \\
\hline \hline
\end{tabular}

appearance of a strong specular neutron spin-flip signal for the second and subsequent reversal, along with the near $90^{\circ}$ angle and full permalloy moment, suggests that the permalloy reversal proceeds via spin rotation involving the majority of ferromagnetic spins with a low dispersion of domain magnetization directions. This stands in contrast to those systems in the literature where the second, and presumably subsequent, reversals occurred by domain wall motion evident in vanishing neutron spin-flip intensity ${ }^{56,57}$ but agrees with the case of rotating domains found in $\mathrm{Co} / \mathrm{CoO} .{ }^{51-53}$ In that case, however, a wider dispersion of angles, ${ }^{53,58}$ along with diffuse scattering, ${ }^{52,58}$ was found to correspond to smaller ferromagnetic domains undergoing incoherent rotation. Figure 15 compares the field dependence and magnitude of the integrated neutron spin-flip signal for the first two reversals across $\mathrm{H}_{0}$ and $\mathrm{H}_{1}$. The neutron spin-flip signal for the first reversal appears in a stepwise fashion, whereas subsequent reversals are symmetric across the coercive field point. This
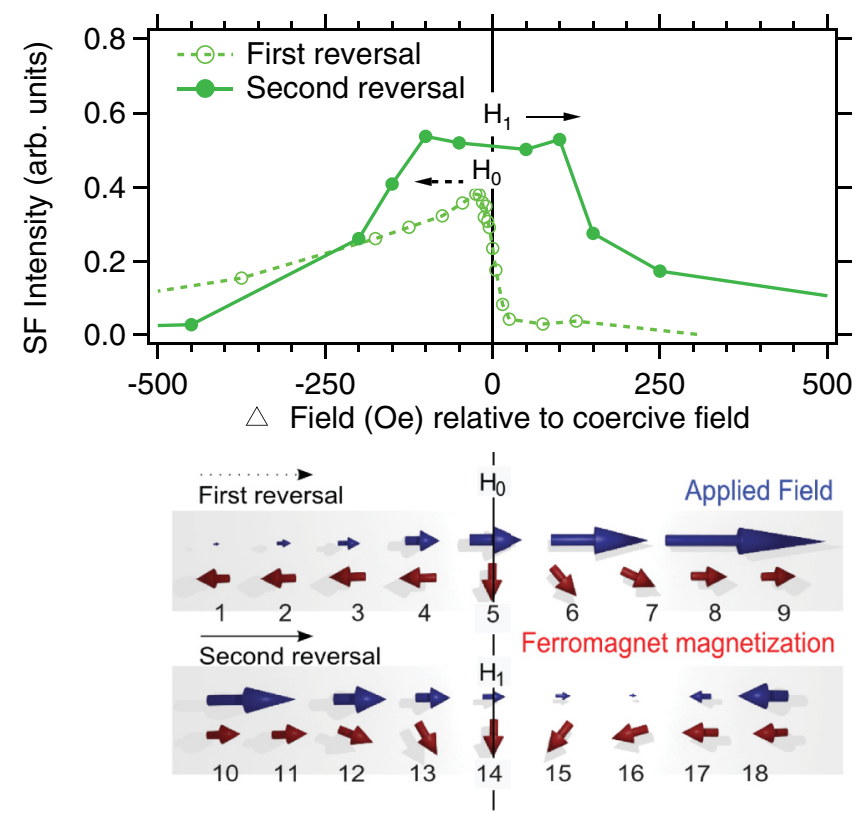

FIG. 15. (Color online) (Top) Integrated neutron spin-flip signal at fields near the coercive fields of first and second reversal points of the untrained loop, $\mathrm{H}_{0}$ and $\mathrm{H}_{1}$. The larger neutron spin-flip intensity for the second reversal indicates a higher degree of coherence in the rotation with less loss of magnetization to domains parallel to the field. (Bottom) Simplified diagram of the sequence leading to the ferromagnetic spin-flop state at the first and second coercive fields $\mathrm{H}_{0}$ and $\mathrm{H}_{1}$. 
suggests an abrupt change in mechanism of reversal on passing through the first coercive field. A weaker neutron spin-flip signal is seen for the first switching point of the untrained loop at $\mathrm{H}_{0}$ with respect to the second reversal at $\mathrm{H}_{1}$. The lower neutron spin-flip signal for the first reversal suggests a higher dispersion in the orientation of ferromagnetic domains, ${ }^{53}$ implying a competition between the motion of domain walls parallel to the field with the rotation of spins within those domains. The non-symmetric reversal mechanism between the first and second switching points is a common feature of exchange bias systems. ${ }^{52,56,58-60}$ In the hematite system, the steplike increase towards a $90^{\circ}$ arrangement of spins during the first reversal-is suggestive of an abrupt spin reorientation of ferromagnetic spins near the coercive field, followed by a slower rotation away from this configuration with increasing fields. This so-called ferromagnetic spin-flop state ${ }^{54}$ was predicted for the $\mathrm{Ni}_{80} \mathrm{Fe}_{20} / \alpha-\mathrm{Fe}_{2} \mathrm{O}_{3}$ system ${ }^{24}$ in accordance with Koon's model, ${ }^{61}$ where a low-energy state could be stabilized with ferromagnetic spins at $90^{\circ}$ to the applied field, as illustrated schematically in the bottom section of Fig. 15. In that work, this was supposed to occur only for specific interface spin structures corresponding to certain crystallite orientations; ${ }^{24}$ however, our experimental result for the polycrystalline hematite film suggests an abrupt transition to this state affecting the majority of ferromagnetic spins during the first reversal, and a growing tendency to linger in this energetically favorable state during subsequent reversals, resulting in coherent rotation for the trained loop. ${ }^{54}$

\section{CONCLUSIONS}

We have provided a comprehensive set of experimental measurements regarding the microstructure and magnetic properties of nanocrystalline permalloy/hematite thin films. These results show conclusively that no significant magnetic impurities or magnetic foreign phases occurred within the bulk of the antiferromagnet and, therefore, eliminate this as a possible explanation for exchange bias. A strong exchange bias for the nanocrystalline case was found below $40 \mathrm{~K}$ and was actually enhanced in magnitude relative to epitaxial films of similar compounds and thicknesses. ${ }^{24}$ The reduced blocking temperature and enhanced exchange bias can be attributed to finite-size and disorder effects for nanostructured hematite. Fitting of the polarized neutron reflectometry patterns show that the data are consistent with an interfacial layer of weakly magnetic spins formed at the antiferromagnetic interface above and below the exchange bias blocking temperature. A detailed error analysis was performed showing that a variety of different interface-depth profiles could describe the data equally well. An asymmetric reversal mechanism was found in the untrained exchange bias state for the descending branch compared to the ascending branch. The reversal mechanism of the trained sample at low temperature was found to be symmetric for both the ascending and descending branches proceeding via an inplane spin rotation. To fully explain the phenomena observed here experimentally for the hematite/permalloy interface, a theoretical framework is required that moves beyond the magnetic properties known for bulk hematite and considers the effect of finite grain-size and temperature-varying anisotropy.

\section{ACKNOWLEDGMENTS}

The research presented herein is made possible by a reflectometer jointly funded by the Canada Foundation for Innovation (CFI), Ontario Innovation Trust (OIT), Ontario Research Fund (ORF), and National Research Council Canada (NRC). The work was partially supported by an Australian Research Council Discovery Project (DP0987190) and the NSC of Taiwan. The access to major research facilities program is supported by the Commonwealth of Australia under the International Science Linkages program. D.L.C acknowledges the support of the Australian Institute Nuclear Science and Engineering and thanks G. McIntyre and A. Nelson for helpful discussions.

\footnotetext{
*dcr@ansto.gov.au

${ }^{\dagger} \mathrm{fk1} @$ ansto.gov.au

${ }^{1}$ A. Morrish, Canted Antiferromagnetism: Hematite (World Scientific, Singapore, 1994).

${ }^{2}$ P. Rochette, P. Mathé, L. Esteban, H. Rakoto, J.-L. Bouchez, Q. Liu, and J. Torrent, Geophys. Res. Lett. 32, L22309 (2005).

${ }^{3}$ R. J. Harrison, S. A. McEnroe, P. Robinson, and C. J. Howard, Am. Mineral. 95, 974 (2010).

${ }^{4}$ I. Dzyaloshinksy, J. Phys. Chem. Solids 4, 241255 (1958).

${ }^{5}$ T. Moriya, Phys. Rev. 120, 91 (1960).

${ }^{6}$ J. Artman, J. Murphy, and S. Foner, Phys. Rev. A 138, 912 (1965).

${ }^{7}$ C. Shull, W. Strauser, and E. Wollan, Phys. Rev. 83, 333 (1951).

${ }^{8}$ M. Reufer, H. Dietsch, U. Gasser, B. Grobety, A. M. Hirt, V. K. Malik, and P. Schurtenberger, J. Phys.: Condens. Matter 23, 065102 (2011).

${ }^{9}$ K. Sreeram, M. Nidhin, and B. Nair, Colloids Surf. B Biointerfaces 71, 260 (2009).

${ }^{10}$ S. Bae, J. H. Judy, D. Fenner, J. Hautala, W. Egelhoff, P. Chen, and L. Gan, J. Magn. Magn. Mater. 320, 2001 (2008).
}

${ }^{11}$ S. Bae, W. Egelhoff, P. J. Chen, and J. H. Judy, IEEE Trans. Magn. 36, 2892 (2000).

${ }^{12}$ H. Sakakima, Y. Sugita, M. Satomi, and Y. Kawawake, J. Magn. Magn. Mater. 198, 9 (1999).

${ }^{13}$ B. Sun, J. Horvat, H. S. Kim, W.-S. Kim, J. Ahn, and G. Wang, J. Phys. Chem. C 114, 18753 (2010).

${ }^{14}$ B. H. Kim and B. I. Min, Phys. Rev. B 77, 094429 (2008).

${ }^{15}$ R. N. Bhowmik and A. Saravanan, J. Appl. Phys. 107, 053916 (2010).

${ }^{16}$ O. Özdemir, D. J. Dunlop, and T. S. Berquó, Geochem. Geophys. Geosyst. 9, 10 (2008).

${ }^{17}$ T. Fujii, M. Takano, R. Kakano, Y. Isozumi, and Y. Bando, J. Magn. Magn. Mater. 135, 231 (1994).

${ }^{18}$ A. H. Hill, F. Jiao, P. G. Bruce, A. Harrison, W. Kockelmann, and C. Ritter, Chem. Mater. 20, 4891 (2008).

${ }^{19}$ R. D. Zysler, D. Fiorani, A. M. Testa, L. Suber, E. Agostinelli, and M. Godinho, Phys. Rev. B 68, 212408 (2003).

${ }^{20}$ S. N. Klausen, K. Lefmann, P. A. Lindgård, L. Theil Kuhn, C. R. H. Bahl, C. Frandsen, S. Mørup, B. Roessli, N. Cavadini, and C. Niedermayer, Phys. Rev. B 70, 214411 (2004). 
${ }^{21}$ S. A. McEnroe, B. Carter-Stiglitz, R. J. Harrison, P. Robinson, K. Fabian, and C. McCammon, Nat. Nano. 2, 631 (2007).

${ }^{22}$ R. Bali, N. Stelmashenko, and M. G. Blamire, J. Appl. Phys. 103, 053911 (2008).

${ }^{23}$ J. van Lierop, K.-W. Lin, Z.-Y. Guo, P.-H. Ko, and H. Ouyang, J. Appl. Phys. 101, 09E505 (2007).

${ }^{24}$ J.Dho, C. W. Leung, Z. H. Barber, and M. G. Blamire, Phys. Rev. B 71, 180402 (2005).

${ }^{25}$ S. Couet, K. Schlage, T. Diederich, R. Rüffer, K. Theis-Bröhl, B. Topervag, K. Zhernenkov, H. Zabel, and R. Röhlsberger, New J. Phys. 11, 013038 (2009).

${ }^{26}$ A. E. Berkowitz, J.-I. Hong, S. K. McCall, E. Shipton, K. T. Chan, T. Leo, and D. J. Smith, Phys. Rev. B 81, 134404 (2010).

${ }^{27}$ W. Cui, W. Liu, X. Zhao, D. Li, and Z. Zhang, J. Alloy. Compd. 475, 42 (2009).

${ }^{28}$ M. James, A. Nelson, S. A. Holt, T. Saerbeck, W. A. Hamilton, and F. Klose, Nucl. Instrum. Methods A 632, 112 (2011).

${ }^{29}$ T. Saerbeck, F. Klose, A. P. Le Brun, J. Füzi, A. Brule, A. Nelson, S. Holt, and M. James, Rev. Sci. Instrum. 83, 081301 (2012).

${ }^{30}$ H. Fritzsche, Rev. Sci. Instrum. 76, 115104 (2005).

${ }^{31}$ A. Nelson, J. Appl. Crystallogr. 39, 273 (2006).

${ }^{32} \mathrm{http} / / / \mathrm{www}-1 \mathrm{lb} . c e a . f r / p r i s m /$ programs/simulreflec/simulreflec.html.

${ }^{33} \mathrm{http} / / /$ rsbweb.nih.gov/ij/ (2011).

${ }^{34}$ S. Bae, J. H. Judy, W. F. Egelhoff, and P. J. Chen, J. Appl. Phys. 87, 6650 (2000).

${ }^{35}$ J. Z. Tischler, J. D. Budai, G. E. Ice, and A. Habenschuss, Acta Crystallogr. Sect. A 44, 22 (1988).

${ }^{36}$ U. Nowak, K. D. Usadel, J. Keller, P. Miltényi, B. Beschoten, and G. Güntherodt, Phys. Rev. B 66, 014430 (2002).

${ }^{37}$ S. Dong, K. Yamauchi, S. Yunoki, R. Yu, S. Liang, A. Moreo, J.-M. Liu, S. Picozzi, and E. Dagotto, Phys. Rev. Lett. 103, 127201 (2009).

${ }^{38}$ A. Hoffmann, J. W. Seo, M. R. Fitzsimmons, H. Siegwart, J. Fompeyrine, J.-P. Locquet, J. A. Dura, and C. F. Majkrzak, Phys. Rev. B 66, 220406 (2002).

${ }^{39}$ S. Roy, M. R. Fitzsimmons, S. Park, M. Dorn, O. Petracic, I. V. Roshchin, Z. P. Li, X. Batlle, R. Morales, A. Misra, X. Zhang, K. Chesnel, J. B. Kortright, S. K. Sinha, and I. K. Schuller, Phys. Rev. Lett. 95, 047201 (2005).

${ }^{40}$ H. Béa, M. Bibes, F. Ott, B. Dupé, X.-H. Zhu, S. Petit, S. Fusil, C. Deranlot, K. Bouzehouane, and A. Barthélémy, Phys. Rev. Lett. 100, 017204 (2008).
${ }^{41}$ M. R. Fitzsimmons, B. J. Kirby, S. Roy, Z.-P. Li, I. V. Roshchin, S. K. Sinha, and I. K. Schuller, Phys. Rev. B 75, 214412 (2007).

${ }^{42}$ S. Roy, C. Sanchez-Hanke, S. Park, M. R. Fitzsimmons, Y. J. Tang, J. I. Hong, D. J. Smith, B. J. Taylor, X. Liu, M. B. Maple, A. E. Berkowitz, C. C. Kao, and S. K. Sinha, Phys. Rev. B 75, 014442 (2007).

${ }^{43}$ S. Brück, G. Schütz, E. Goering, X. Ji, and K. M. Krishnan, Phys. Rev. Lett. 101, 126402 (2008).

${ }^{44}$ P. Kappenberger, S. Martin, Y. Pellmont, H. J. Hug, J. B. Kortright, O. Hellwig, and E. E. Fullerton, Phys. Rev. Lett. 91, 267202 (2003).

${ }^{45}$ C. E. ViolBarbosa, H. L. Meyerheim, E. Jal, J.-M. Tonnerre, M. Przybylski, L. M. Sandratskii, F. Yildiz, U. Staub, and J. Kirschner, Phys. Rev. B 85, 184414 (2012).

${ }^{46}$ E. Fulcomer and S. H. Charap, J. Appl. Phys. 43, 4190 (1972).

${ }^{47}$ K. O’Grady, J. Phys. D 322, 883 (2010).

${ }^{48}$ S. H. Chung, A. Hoffmann, and M. Grimsditch, Phys. Rev. B 71, 214430 (2005).

${ }^{49}$ M. Kiwi, J. Magn. Magn. Mater. 234, 584 (2001).

${ }^{50}$ H. Chow and P. Feffer, Phys. Rev. B 10, 243 (1974).

${ }^{51}$ M. Gierlings, M. J. Prandolini, H. Fritzsche, M. Gruyters, and D. Riegel, Phys. Rev. B 65, 092407 (2002).

${ }^{52}$ F. Radu, M. Etzkorn, R. Siebrecht, T. Schmitte, K. Westerholt, and H. Zabel, Phys. Rev. B 67, 134409 (2003).

${ }^{53}$ W.-T. Lee, S. G. E. te Velthuis, G. P. Felcher, F. Klose, T. Gredig, and E. D. Dahlberg, Phys. Rev. B 65, 224417 (2002).

${ }^{54}$ A. Hoffmann, Phys. Rev. Lett. 93, 097203 (2004).

${ }^{55}$ P. Blomqvist, K. M. Krishnan, and H. Ohldag, Phys. Rev. Lett. 94, 107203 (2005)

${ }^{56}$ M. R. Fitzsimmons, P. Yashar, C. Leighton, I. K. Schuller, J. Nogues, C. F. Majkrzak, and J. A. Dura, Phys. Rev. Lett. 84, 3986 (2000).

${ }^{57}$ C. Leighton, M. R. Fitzsimmons, P. Yashar, A. Hoffmann, J. Nogues, J. Dura, C. F. Majkrzak, and I. K. Schuller, Phys. Rev. Lett. 86, 4394 (2001).

${ }^{58}$ M. Gierlings, M. Prandolini, H. Fritzsche, M. Gruyters, and D. Riegel, Physica B 356, 36 (2005).

${ }^{59}$ A. Paul, E. Kentzinger, U. Rücker, and T. Brückel, Phys. Rev. B 73, 092410 (2006).

${ }^{60}$ A. Paul, E. Kentzinger, U. Rücker, D. E. Bürgler, and P. Grünberg, Phys. Rev. B 70, 224410 (2004).

${ }^{61}$ N. C. Koon, Phys. Rev. Lett. 78, 4865 (1997). 